\title{
N-Alkylimidazole-Decorated Dendritic Catalysts for Baylis-Hillman Reaction
}

Baylis-Hillman

reaction

N-alkylated imidzoles

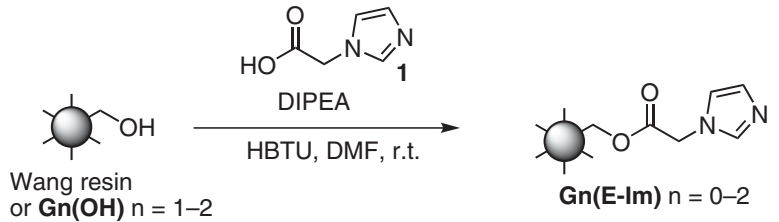

dendrons

heterogeneous

catalysis

or $\mathrm{Gn}(\mathrm{OH}) \mathrm{n}=1-2$

Gn(E-Im) $n=0-2$

HBTU = tetramethyl- $\mathrm{O}-(1 \mathrm{H}$-benzotriazol-1-yl)uronium hexafluorophosphate
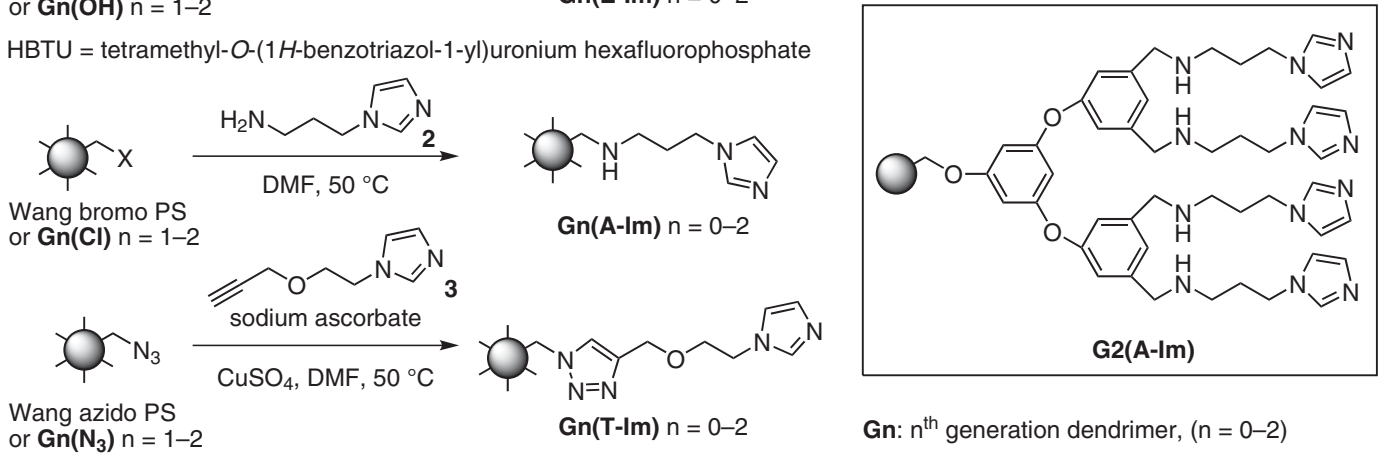

SYNFACTIth or $\mathbf{G n}\left(\mathrm{N}_{3}\right) \mathrm{n}=1-2$

$\mathrm{Gn}(\mathrm{T}-\mathrm{Im}) \mathrm{n}=0-2$

Gn: $\mathrm{n}^{\text {th }}$ generation dendrimer, $(\mathrm{n}=0-2)$<smiles>CC(C)=CC(=O)OC=O</smiles>

(3 mol equiv)

G0(E-Im): $11 \%$ yield; DMF, $48 \mathrm{~h}$ $31 \%$ yield; DMF- $\mathrm{H}_{2} \mathrm{O}(9: 1), 48 \mathrm{~h}$ G1(E-Im): 43\% yield; DMF- $\mathrm{H}_{2} \mathrm{O}(9: 1), 48 \mathrm{~h}$ G2(E-Im): 59\% yield; DMF- $\mathrm{H}_{2} \mathrm{O}$ (9:1), $48 \mathrm{~h}$

G0(T-Im): 6\% yield; DMF, $22 \mathrm{~h}$

$58 \%$ yield; DMF- $\mathrm{H}_{2} \mathrm{O}(9: 1), 22 \mathrm{~h}$

G1 (T-Im): 74\% yield; DMF- $\mathrm{H}_{2} \mathrm{O}(9: 1), 22 \mathrm{~h}$

G2(T-Im): 100\% yield; DMF- $\mathrm{H}_{2} \mathrm{O}(9: 1), 22 \mathrm{~h}$

\section{$\underset{\text { solvent, time }}{\stackrel{\text { catalyst }}{\longrightarrow}}$}

GO(A-Im): $34 \%$ yield: DMF- $\mathrm{H}_{2} \mathrm{O}(9: 1), 4 \mathrm{~h}$ $69 \%$ yield; $\mathrm{DMF}-\mathrm{H}_{2} \mathrm{O}(8: 2), 6 \mathrm{~h}$ $38 \%$ yield; DMF- $\mathrm{H}_{2} \mathrm{O}(7: 3), 1.5 \mathrm{~h}$ $97 \%$ yield; DMF- $\mathrm{H}_{2} \mathrm{O}(1: 1), 6 \mathrm{~h}$ $76 \%$ yield; $\mathrm{THF}-\mathrm{H}_{2} \mathrm{O}(9: 1), 6 \mathrm{~h}$

G1 $(\mathrm{A}-\mathrm{Im}): 47 \%$ yield; DMF- $\mathrm{H}_{2} \mathrm{O}(9: 1), 4 \mathrm{~h}$ $62 \%$ yield; DMF- $\mathrm{H}_{2} \mathrm{O}(9: 1), 1.5 \mathrm{~h}$ $95 \%$ yield; $\mathrm{THF}-\mathrm{H}_{2} \mathrm{O}(9: 1), 6 \mathrm{~h}$

G2(A-Im): 91\% yield; DMF- $\mathrm{H}_{2} \mathrm{O}(9: 1), 4 \mathrm{~h}$ $95 \%$ yield; DMF- $\mathrm{H}_{2} \mathrm{O}(7: 3), 1.5 \mathrm{~h}$ $100 \%$ yield; $\mathrm{THF}-\mathrm{H}_{2} \mathrm{O}(9: 1), 6 \mathrm{~h}$
Significance: Polymer-supported dendritic catalysts [Gn(E-Im), Gn(A-Im), Gn(T-Im)] were prepared from Wang resins and $\mathrm{N}$-alkylated imidazoles (1-3), and applied to the Baylis-Hillman reaction. Thus, the Baylis-Hillman reaction of methyl vinyl ketone and 4-nitrobenzaldehyde was performed with the catalytic resin G2(A-Im) $0.1 \mathrm{mmol}$ of $\mathrm{N}$-alkylated imidazole units) in DMF- $\mathrm{H}_{2} \mathrm{O}(9: 1$, $\mathrm{v} / \mathrm{v}$ ) at room temperature for $4 \mathrm{~h}$ to afford adduct 4 in $91 \%$ yield.
Comment: The addition of water improved the catalytic activity in the Baylis-Hillman reaction. The second-generation catalysts [G2(E-Im), G2(T-Im), G2(A-Im)] exhibited higher catalytic activity than their non-dendritic counterparts [GO(EIm), GO(T-Im), GO(A-Im)]. 